\title{
Triangular Scheme Revisited in the Light of $n$-permutable Categories
}

\author{
Brice Réné Amougou Mbarga
}

University of Yaoundé I, Laboratory of Algebra, Geometry and Application, P.O.Box: 812, Yaoundé, Cameroon; e-mail: renebrice3@gmail.com

\begin{abstract}
The first diagrammatic scheme was developed by H.P. Gumm under the name Shifting Lemma in case to characterize congruence modularity. A diagrammatic scheme is developed for the generalized semi distributive law in Mal'tsev categories. In this paper we study this diagrammatic scheme in the context of $n$-permutable, and of Mal'tsev categories in particular. Several remarks concerning the Triangular scheme case are included.
\end{abstract}

\section{Introduction}

We are going to establish a diagrammatic scheme for equivalence distributivity in equivalence $n$-permutable category. In this section we recall some basic definitions and results from the literature, needed throughout the article.

\section{$1.1 n$-permutable varieties}

A variety of universal algebras is called $n$-permutable, $n \geq 2$, when any pair of congruences $R$ and $S$ on a same algebra $n$-permutes: $(R, S)_{n}=(S, R)_{n}$. Where $(R, S)_{n}$ denotes the composite $R S R S \cdots$ of $R$ and $S, n$ times. This notion determines a sequence of families of varieties, whose first two instances are well known: for $n \geq 2$, we regain 2-permutable varieties [15], better known as Mal'tsev varieties; for $n \geq 3$,

Received: October 9, 2020; Revised: December 16, 2020; Accepted: December 19, 2020

2010 Mathematics Subject Classification: 08C05.

Keywords and phrases: categories, $n$-permutable, triangular scheme. 
these are the 3-permutable varieties. The property of $n$-permutability makes sense in any regular category and was generalised to this categorical context in [5], where $n$-permutable categories were first studied. A variety is a Mal'tsev variety precisely when its theory contains a ternary operation $p$ such that the identities $p(x, y, y)=x$ and $p(x, x, y)=y$ hold [14]. 3-permutable varieties are characterised by the existence of two ternary operations $r$ and $s$ satisfying the identities $r(x, y, y)=x, r(x, x, y)=s(x, y, y)$ and $s(x, x, y)=y$. Equivalently, they are characterised by the existence of quaternary operations $p$ and $q$ such that the identities $p(x, y, y, z)=x, p(x, x, y, y)=q(x, x, y, y)$ and $q(x, y, y, z)=z$ hold. Congruence distributive varieties were characterized by Jónsson [18] by means of the Maltsev condition. A lattice $L$ is called distributive when

$$
b \wedge(a \vee c)=(b \wedge a) \vee(b \wedge c) .
$$

Equivalently, $L$ is distributive if and only if it satisfies the Horn sentence

$$
a \wedge b \leq c \Rightarrow b \wedge(a \vee b) \leq c .
$$

Hence, $(s)$ is a Horn sentence and a lattice $L$ satisfies $(d)$ if and only if it satisfies $(s)$. Thus $(s)$ is another characterization of distributivity. A variety $\mathcal{V}$ of universal algebras is called congruence distributive when the lattice Cong(A) of congruences on any algebra $A$ in $\mathcal{V}$ is distributive. Recall that a lattice $L$ is $\wedge$-semidistributive if

$$
y \wedge x=y \wedge z \Rightarrow y \wedge(x \vee z)=y \wedge z \text { for all } x, y, z \in L .
$$

The $\wedge$-semidistributive law above is often denoted by $S D_{\wedge}$. More general (in fact, weaker) Horn sentences have been investigated in Geyer [12]. For $n \geq 2$ put $\underline{n}=$ $\{0,1, \cdots, n-1\}$ and let $l_{2}(\underline{n})$ denote the set $\{I: I \subseteq \underline{n}$ and $|I| \geq 2\}$. For $\emptyset \neq K \subseteq l_{2}(\underline{n})$ we define the generalized meet semidistributive law $S D_{\wedge}(n, K)$ for lattices as follows: for all $x, y_{0}, \cdots, y_{n-1}$

$$
x \wedge y_{0}=x \wedge y_{1}=\cdots=x \wedge y_{n-1} \Rightarrow x \wedge y_{0}=\bigwedge_{J \in K} \bigvee_{j \in J} y_{j}
$$

As a particular case, when $K=\{J: J \subseteq \underline{n}$ and $|J|=2\}$ is denoted by $S D_{\wedge}(n, 2)$. Notice that $S D_{\wedge}(n, 2)$ is the following lattice Horn sentence:

$$
x \wedge y_{0}=x \wedge y_{1}=\cdots=x \wedge y_{n-1} \Rightarrow x \wedge y_{0}=x \wedge \bigwedge_{0 \leq i<j<n}\left(y_{i} \vee y_{j}\right) .
$$


In particular, $S D_{\wedge}(2,2)$ is the $\wedge$-semidistributivity law defined in by:

$$
x \wedge y=y \wedge z \Rightarrow y \wedge(x \vee z)=y \wedge z \text { for all } x, y, z \in L . \quad\left(S D_{\wedge}\right)
$$

\section{$1.2 n$-schemes and weak $n$-scheme}

Recall from [16] that a sublattice $L$ of an equivalence lattice $E q A$ satisfies the Triangular scheme if for each $R, S, T \in L$ with $R \wedge S \subseteq T$ and for $x, y, z \in A$ such that $\langle x, y\rangle \in T$, $\langle x, z\rangle \in S,\langle z, y\rangle \in R$ we have $\langle z, y\rangle \in T$.

This can be visualized as follows:
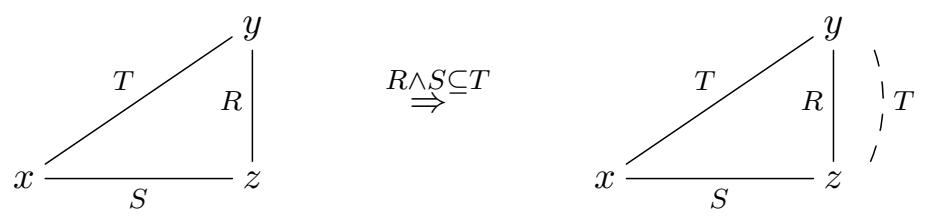

The following assertions are proved in [17]:

(a) if $\operatorname{Con} A$ is distributive, then it satisfies the Triangular scheme;

(b) if $A$ is a congruence permutable algebra, then $\operatorname{Con} A$ is distributive if and only if Con $A$ satisfies the Triangular scheme.

We are going to establish a diagrammatic scheme for equivalence distributivity in equivalence $n$-permutable category. The scheme is similar to that of Gumm [26] for congruence modularity.

A sublattice $L$ of $E q A$ satisfies the $n$-scheme (the weak $n$-scheme) if for each $R, S, T \in L$ with $R \wedge S \subseteq T$ (or $R \wedge S=R \wedge T$, respectively) and for $x, y, z_{1}, \cdots, z_{n} \in$ $A$ such that

$$
\langle x, y\rangle \in R,\left\langle x, z_{1}\right\rangle \in S,\left\langle z_{1}, z_{2}\right\rangle \in T,\left\langle z_{2}, z_{3}\right\rangle \in S, \cdots,\left\langle z_{n-1}, y\right\rangle \in S
$$

for $n$ odd and $\left\langle z_{n-1}, y\right\rangle \in T$ for $n$ even we have $\langle x, y\rangle \in T$. These schemes can be also visualized but, contrary to the previous cases, classes of the same congruence fail to be 
parallel:
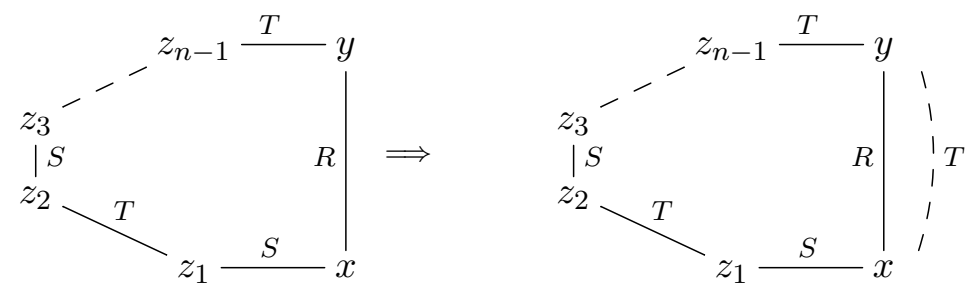

\subsection{Regular categories and relations}

Our categories will always be regular, in the sense of Barr [1]; we recall that a category is regular if it has finite limits, each arrow factors as a regular epi followed by a mono, and regular epis are pull-back stable. (It turns out that in a regular category the kernel pair of an arrow always has a coequalizer, given by the regular epi part of the factorization of the arrow). In a regular category, it is possible to compose relations. If $\left(R, r_{1}, r_{2}\right)$ is a relation from $X$ to $Y$ and $\left(S, s_{1}, s_{2}\right)$ a relation from $Y$ to $Z$, their composite $S R$ is a relation from $X$ to $Z$ obtained as the regular image of the arrow

$$
\left(r_{1} \pi_{1}, s_{2} \pi_{2}\right): R \times_{Y} S \longrightarrow X \times Z
$$

where $\left(R \times_{Y} S, \pi_{1}, \pi_{2}\right)$ is the pullback of $r_{2}$ along $s_{1}$. The composition of relations is then associative, thanks to the fact that regular epimorphisms are assumed to be pullback stable.

\section{Regular Mal'tsev Categories and Triangular Scheme}

A finitely complete category $\mathcal{E}$ is called a Mal'tsev category if every reflexive relation in $\mathcal{E}$ is an equivalence relation. These categories are also characterized by other properties on relations, as follows:

Theorem 2.1. [16] Let $\mathcal{E}$ be a regular category. Then the following statements are equivalent:

(i) $\mathcal{E}$ is a Mal'tsev category; 
(ii) $\forall F, E \in E q u i v(X), E \vee F=F E(=E F) \in E q u i v(X)$, for any object $X$;

(iii) every reflexive relation $E$ in $\mathcal{E}$ is symmetric: $E^{\circ}=E$.

Theorem 2.2. Let $\mathcal{E}$ be a regular Mal'tsev category. Then the following conditions are equivalent:

(1) the Triangular scheme holds in $\mathcal{E}$;

(2) $\forall E, F, P \in E q u i v(X)$ we have

$$
F \wedge(E P) \subseteq P \vee(E \wedge F)
$$

(3) $\forall E, F, P \in E q u i v(X)$ then

$$
F \wedge(E P) \subseteq(E \vee(F \wedge P)) \wedge(P \vee(E \wedge F))
$$

Proof. To prove the implication $(1) \Rightarrow(2)$ suppose $E, F, P \in E q u i v(X)$ are arbitrary, and $(c, a) \in F \wedge(E P)$. Then there exists an element $b$ such that $a P b E c$. Apply the triangular scheme for these elements with $P$ replaced by $P^{\prime}=P \vee(E \wedge F)$. We get that

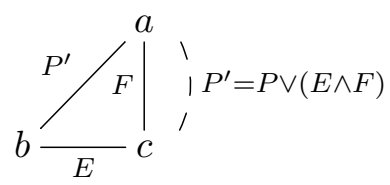

Now if (2) is true, then reversing the roles of $E$ and $P$ we get that

$$
F \wedge(P E) \subseteq E \vee(P \vee F)
$$

By taking the converse of this inclusion and combining with (2) we obtain condition (3). Therefore the implication $(2) \Rightarrow(3)$ is proved. (3) $\Rightarrow(2)$ Obvious.

(2) $\Rightarrow(1)$ Let $R, S$ and $T$ be equivalence relations on an object $X$ such that $R \wedge S \leq$ $T$ we show that $R \wedge S T \leq T$.

We have

$$
R \wedge S T \leq T \vee(S \wedge R) \leq T \vee T=T .
$$


Proposition 2.3. Let $\mathcal{E}$ be a regular Mal'tsev category. Then the following conditions are equivalent:

(1) Equiv $(X)$ satisfies $S D_{\wedge}(n, 2)$ for $X$ in $\mathcal{E}$;

(2) the scheme depicted in Figure 1 holds for $S, R_{0}, \cdots, R_{n-1}$ in Equiv $(X)$ and $x_{0}, \cdots, x_{k}, y, z$, are related as in Figure 1 where $k=\frac{n(n-1)}{2}-1$ and $T$ stands for $R_{0} \wedge R_{1} \wedge \cdots \wedge R_{n-1}$.

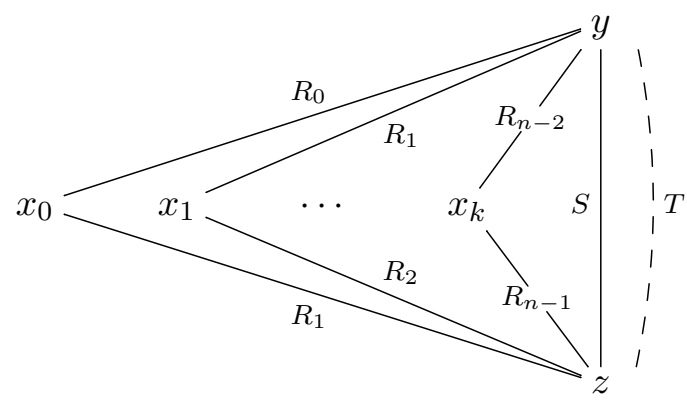

Figure 1

Proof. Suppose $S D_{\wedge}(n, 2)$ holds. Using the premise of $S D_{\wedge}(n, 2)$ we obtain

$$
S \wedge R_{0}=\left(S \wedge R_{0}\right) \wedge \cdots \wedge\left(S \wedge R_{0}\right)=S \wedge\left(R_{0} \wedge \cdots \wedge R_{n-1}\right) \subseteq T
$$

whence $\operatorname{Equiv}(X)$ satisfies the Horn sentence

$$
S \wedge R_{0}=S \wedge R_{1}=\cdots=S \wedge R_{n-1} \Rightarrow S \wedge \bigwedge_{0 \leq i<j<n}\left(R_{i} \vee R_{j}\right) \leq T .
$$

This implies the scheme, for the situation on the left hand side in Figure 1 then gives $(y, z) \in S \wedge \bigwedge_{0 \leq i<j<n}\left(R_{i} \circ R_{j}\right) \leq T$. To show the converse, suppose that the scheme given by Figure 1 holds, $S, R_{0}, \cdots, R_{n-1}$ in $\operatorname{Equiv}(X)$ with $S \wedge R_{0}=S \wedge R_{1}=\cdots=$ $S \wedge R_{n-1}$, and suppose that $(y, z) \in S \wedge \bigwedge_{0 \leq i<j<n}\left(R_{i} \circ R_{j}\right)$, there exist $x_{0}, x_{1}, \cdots, x_{k}$ of $X$ such that for each $j(1 \leq j \leq k)$ there exist $u, v$ such that $\left(z, x_{j}\right) \in R_{u}$ and $\left(x_{j}, y\right) \in R_{v}$ (according to the left hand side of Figure 1). Then the scheme applies and we conclude $(y, z) \in T$. Since $T \subseteq R_{0},(y, z) \in R_{0}$. Hence $(y, z) \in S \wedge R_{0}$. This proves the " $\leq "$ 
part of $S D_{\wedge}(n, 2)$. The reverse part is simpler and does not need the scheme: $S \supseteq S \wedge R_{0}$ and $R_{i} \vee R_{j} \supseteq R_{j} \supseteq S \wedge R_{i}=S \wedge R_{0}$ clearly give $S \wedge \bigwedge_{0 \leq i<j<n}\left(R_{i} \vee R_{j}\right) \supseteq S \wedge R_{0}$ proving the theorem.

Note that, for diagram such as Figure 1 where $R_{i}, S$ or $T$ are not symmetric, the relations are always to be considered from left to right and from top to bottom. To avoid ambiguity with the interpretation of such diagrams, from now on we will write $a \stackrel{E}{\longrightarrow} b$ to mean that $(a, b) \in E$ whenever $E$ is a non-symmetric relation.

Theorem 2.4. Let $\mathcal{E}$ be a regular category. Then the following conditions are equivalent:

(i) $\mathcal{E}$ is an equivalence $S D_{\wedge}(n, 2)$ Mal'tsev category;

(ii) the scheme depicted in Figure 1 holds when $S, R_{0}, \cdots, R_{n-1}$ and $T$ are reflexive relations.

Proof. $(i) \Rightarrow$ (ii) Since $\mathcal{E}$ is a Mal'tsev category, reflexive relations are necessarily equivalence relations. Since $\mathcal{E}$ is also equivalence $S D_{\wedge}(n, 2)$, by Proposition 2.3 , the the scheme depicted in Figure 1 holds for any reflexive relations in $\mathcal{E}$.

$($ ii $) \Rightarrow(i)$ To prove that $\mathcal{E}$ is a Mal'tsev category, we show that any reflexive relation $\left\langle e_{1}, e_{2}\right\rangle: E \longmapsto X \times X$ in $\mathcal{E}$ is also symmetric (Theorem 2.1(iii)). Suppose that $(x, y) \in$ $E$, and consider the reflexive relations $T$ and $S$ on $E$ defined as follows:

$$
(a E b, c E d) \in S \Leftrightarrow(a, d) \in E \text {, and }(a E b, c E d) \in T \Leftrightarrow(c, b) \in E .
$$

Let $R_{i}=T$, for all $1 \leq i \leq n-1$.

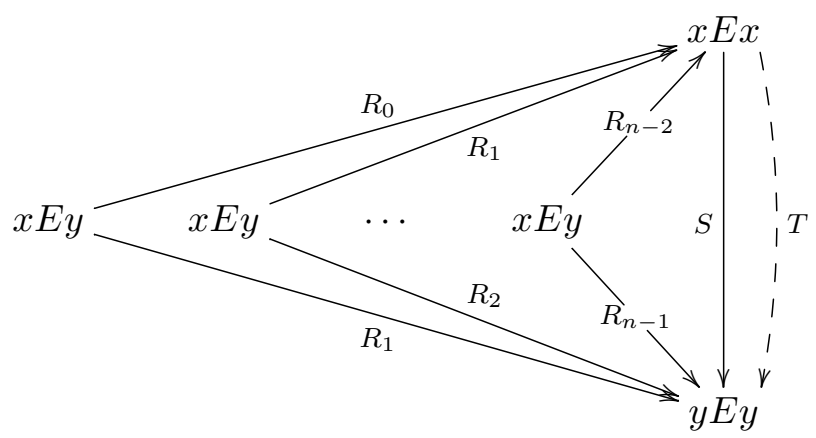


( $x E x$ and $y E y$ by the reflexivity of the relation $E$ ). We conclude that $(x E x, y E y) \in$ $T$ and, consequently, that $(y, x) \in E$, so that $\mathcal{E}$ is a Mal'tsev category. Since the Figure 1 holds in $\mathcal{E}$, by Proposition 2.3 the category $\mathcal{E}$ is equivalence $S D_{\wedge}(n, 2)$.

\section{$3 n$-permutable category}

Definition 3.1. [23] A regular category $\mathcal{E}$ is an a $n$ permutable category when the composition of (effective) equivalence relations on a given object is $n$-permutable: for two (effective) equivalence relations $R$ and $S$ on the same object, we have $(R, S)_{n}=$ $(S, R)_{n}$. Where the composition of $n$ alternating factors $R$ and $S$ is denoted by $(R, S)_{n}=R S R S \cdots$

Theorem 3.2. ([23], Theorem 3.5 of [5]) Let $n \geq 2$ and let $\mathcal{E}$ be a regular category. Then the following statements are equivalent:

(i) $\mathcal{E}$ is n-permutable category;

(ii) $\left(P, P^{\circ}\right)_{n+1} \leq\left(P, P^{\circ}\right)_{n-1}$ for any relation $P$;

(iii) $(R, S)_{n}$ is an equivalence relation and is therefore $R \vee S$;

(iv) $\left(1_{X} \wedge T\right) T^{\circ}\left(1_{X} \wedge T\right) \leq T^{n-1}$, for any relation $T$ on an objet $X$;

(v) for any reflexive endorelation $E \longmapsto X \times X$ in $\mathcal{E}$, the relation $\left(E, E^{\circ}\right)_{n-1}$ is an equivalence relation;

(vi) for any such reflexive endorelation $E$, the relation $\left(E, E^{\circ}\right)_{n-1}$ is transitive;

(vii) for any such reflexive endorelation $E$ we have $\left(E, E^{\circ}\right)_{n-1}=\left(E^{\circ}, E\right)_{n-1}$;

(viii) $E^{\circ} \leq E^{n-1}$ for any reflexive relation $E$.

Theorem 3.3. Let $\mathcal{E}$ be n-permutable category. Then

(i) $\mathcal{E}$ is an equivalence $\wedge$-semidistributive if and only if it satisfies the weak $n$-scheme.

(ii) $\mathcal{E}$ is an equivalence distributive if and only if it satisfies the $n$-scheme. 
Proof. For (i), suppose $R, S, T$ in $\operatorname{Equiv}(X)$ with $R \wedge S=R \wedge T$ and $x, y, z_{1}, \cdots, z_{n-1} \in X$ such that the assumptions of the weak scheme are satisfied. Then

$$
\langle x, y\rangle \in R \wedge(S, T)_{n}=R \wedge(S \vee T)=R \wedge T
$$

due to $\wedge$-semidistributivity, i.e. it satisfies the weak $n$-scheme for each $n \geq 2$. Conversely, let $\operatorname{Equiv}(\mathrm{X})$ satisfy the weak $n$-scheme, let $R, S, T$ in $\operatorname{Equiv}(X)$ with $R \wedge S=R \wedge T$. Let $\langle x, y\rangle \in R \wedge(S \vee T)$. Due to equivalence $n$-permutability, we have $\langle x, y\rangle \in R \wedge(S, T)_{n}$ with $n$ factors. Thus it is almost evident that the assumptions of the weak n-scheme are satisfied. Applying this scheme, we conclude $\langle x, y\rangle \in R \wedge T$. We have shown $R \wedge(S \vee T) \subseteq R \wedge T$.

(ii) Obvious.

Let $n$ be an odd number $(n \geq 3)$. A relation $P \longmapsto X \times X$ on $X$ is called positive when it is of the form $P=\left(E^{\circ}, E\right)_{n-1}$, for some relation $E \longmapsto X \times X$. In set-theoretic terms, $P$ is positive when there exists a relation $E$ and $x_{1}, x_{2}, \cdots, x_{n-1}$ such that $\left(x, x^{\prime}\right) \in P$ if $\left(x, x_{1}\right) \in E,\left(x_{2}, x_{1}\right) \in E \cdots\left(x^{\prime}, x_{n-2}\right) \in E$.

Proposition 3.4. Let $n$ be an odd number $(n \geq 3)$. A regular category $\mathcal{E}$ is an $n$-permutable category if and only if any reflexive and positive relation in $\mathcal{E}$ is an equivalence relation.

Proof. Suppose that $\mathcal{E}$ is an $n$-permutable category and consider a reflexive and positive relation $P$ with $1 \leq P=\left(E^{\circ}, E\right)_{n-1}$. Then $P$ is symmetric. Since

$$
P^{\circ}=\left(E, E^{\circ}\right)_{n-1}=\left(E^{\circ}, E\right)_{n+1}=\left(E^{\circ}, E\right)_{n-1} .
$$

And As for the transitivity of $P$, we have

$$
\left(E^{\circ}, E\right)_{n-1}\left(E^{\circ}, E\right)_{n-1}=\left(E^{\circ}, E\right)_{2 n-2}=\left(E^{\circ}, E\right)_{n-1} .
$$

By Theorem $3.2(i) \Rightarrow(i i)$. Conversely, let $U$ be a reflexive relation on $X$. Then $P=\left(U^{\circ}, U\right)_{n-1}$ is a reflexive and positive relation, thus an equivalence relation by assumption. It follows that $\mathcal{E}$ is an $n$-permutable category by Theorem 3.2 (v). 


\section{References}

[1] M. Barr, Exact categories, in: Exact Categories and Categories of Sheaves, 1-120, Lecture Notes in Math., 236, Springer, Berlin, 1971.

[2] F. Borceux and D. Bourn, Mal'cev, protomodular, homological and semi-abelian categories, Mathematics and its Applications 566, Kluwer Academic Publishers, Dordrecht, 2004.

[3] S. Burris and H.P. Sankappanavar, A course in universal algebra, Graduate Texts in Mathematics, 78, Springer-Verlag, New York-Berlin, 1981.

[4] A. Carboni, J. Lambek and M.C. Pedicchio, Diagram chasing in Mal'cev categories, Appl. Algebra 69 (1990), 271-284.

[5] A. Carboni, G.M. Kelly and M.C. Pedicchio, Some remarks on Mal'tsev and Goursat categories, Appl. Categ. Structures 1 (1993), 385-421.

[6] I. Chajda and R. Halas, On schemes for congruence distributivity, Open Mathematics 2(3) (2004), 368-376. https://doi.org/10.2478/BF02475233

[7] I. Chajda, A note on the triangular scheme, East-West J. Math. 3 (2001), 79-80.

[8] I. Chajda and E.K. Horváth, A triangular scheme for congruence distributivity, Acta Sci. Math. (Szeged) 68 (2002), 29-35.

[9] I. Chajda and E.K. Horváth, A scheme for congruence semidistributivity, Discuss. Math. Gen. Algebra Appl. 23 (2003), 13-18.

[10] I. Chajda, E.K. Horváth and G. Czédli, Trapezoid lemma and congruence distributivity, Math. Slovaca 53 (2003), 247-253.

[11] J. Duda, The Triangular Principle for congruence distributive varieties, Abstract of a seminar lecture presented in Brno, March, 2000.

[12] W. Geyer, Generalizing semidistributivity, Order 10 (1903), 77-92.

[13] M. Gran, Notes on regular, exact and additive categories, Summer School on Category Theory and Algebraic Topology, Ecole Polytechnique Fédérale de Lausanne, 11-13 September 2014. 
[14] M. Gran and D. Rodelo, A new characterisation of Goursat categories, Appl. Categ. Structures 20 (2012), 229-238. doi:10.1007/s10485-010-9236-x

[15] M. Gran and D. Rodelo, Beck-chavalley condition and Goursat categories, 2013. arxiv:1512.04066v1

[16] M. Gran, D. Rodelo and I. Tchoffo Nguefeu, Variations of the shifting lemma and Goursat categories, Algebra Universalis 80 (2018), Paper No. 2, 12 pp.

[17] M. Gran, D. Rodelo and I. Tchoffo Nguefeu, Facets of congruence distributivity in Goursat categories, 2020. arXiv:1909.10211v2

[18] B. Jonsson Algebras whose congruence lattices are distributive, Math. Scand. 21 (1967), 110-121.

[19] J. Hagemann and A. Mitschke, On n-permutable congruences, Algebra Universalis 3 (1973), 8-12.

[20] M. Hoefnagel, Majority categories, Theory Appl. Categories 34 (2019), 249-268.

[21] M. Hoefnagel, Characterizations of majority categories, Appl. Categ. Structures 28 (2020), 113-134.

[22] M. Hoefnagel, A categorical approach to lattice-like structures, Ph.D. thesis, 2018.

[23] P.-A. Jacqmin and D. Rodelo, Stability properties characterising $n$-permutable categories, Theory Appl. Categ. 32 (2017), Paper No. 45, 1563-1587.

[24] K.A. Kearnes and E.W. Kiss, The triangular principle is equivalent to the triangular scheme, Algebra Universalis 54 (2005), 373-383. https://doi.org/10.1007/s00012-005-1954-9

[25] H. Peter Gumm, Geometrical methods in congruence modular algebras, Mem. Amer. Math. Soc. 45 (1983).

[26] H. Peter Gumm, The little Desarguesian theorem for algebras in modular varieties, Proc. Amer. Math. Soc. 80(3) (1980), 393-397.

[27] D. Rodelo and T. Van der Linden, Approximation hagemann-Mitschke co-operations, Appl. Categ. Structures 22 (2014), 1009-1020. 
[28] T. Van der Linden and C. Sandry Simeu, Higher extensions in exact Mal'tsev categories: distributivity of congruences and the $3^{n}$-lemma, 2018. arXiv:1807.03164v1

This is an open access article distributed under the terms of the Creative Commons Attribution License (http://creativecommons.org/licenses/by/4.0/), which permits unrestricted, use, distribution and reproduction in any medium, or format for any purpose, even commercially provided the work is properly cited. 